\title{
Lorentzian noise in the two-dimensional electron gas of AlxGa1-XAs/GaAs quantum wells
}

\section{Citation for published version (APA):}

Chen, Y., Vliet, van, C. M., Koenraad, P. M., \& Larkins Jr., G. L. (1999). Lorentzian noise in the two-dimensional electron gas of AlxGa1-xAs/GaAs quantum wells. Journal of Applied Physics, 86(11), 6206-6212.

https://doi.org/10.1063/1.371732

DOI:

10.1063/1.371732

Document status and date:

Published: 01/01/1999

\section{Document Version:}

Publisher's PDF, also known as Version of Record (includes final page, issue and volume numbers)

\section{Please check the document version of this publication:}

- A submitted manuscript is the version of the article upon submission and before peer-review. There can be important differences between the submitted version and the official published version of record. People interested in the research are advised to contact the author for the final version of the publication, or visit the $\mathrm{DOI}$ to the publisher's website.

- The final author version and the galley proof are versions of the publication after peer review.

- The final published version features the final layout of the paper including the volume, issue and page numbers.

Link to publication

\section{General rights}

Copyright and moral rights for the publications made accessible in the public portal are retained by the authors and/or other copyright owners and it is a condition of accessing publications that users recognise and abide by the legal requirements associated with these rights.

- Users may download and print one copy of any publication from the public portal for the purpose of private study or research.

- You may not further distribute the material or use it for any profit-making activity or commercial gain

- You may freely distribute the URL identifying the publication in the public portal.

If the publication is distributed under the terms of Article 25fa of the Dutch Copyright Act, indicated by the "Taverne" license above, please follow below link for the End User Agreement:

www.tue.nl/taverne

Take down policy

If you believe that this document breaches copyright please contact us at:

openaccess@tue.nl

providing details and we will investigate your claim. 


\title{
Lorentzian noise in the two-dimensional electron gas of $\mathrm{Al}_{x} \mathrm{Ga}_{1-x} \mathrm{As} / \mathrm{GaAs}$ quantum wells
}

\author{
Yuping Chen and Carolyne M. Van Vliet ${ }^{\mathrm{a})}$ \\ Center for Engineering and Applied Sciences, Florida International University, Miami, Florida 33174
}

Paul M. Koenraad

Department of Physics, Eindhoven University of Technology, P.O. Box 513, 5600 MB Eindhoven, The Netherlands

Grover L. Larkins, Jr. Center for Engineering and Applied Sciences, Florida International University, Miami, Florida 33174

(Received 14 June 1999; accepted for publication 2 September 1999)

\begin{abstract}
Current noise spectra $S_{I}(\omega)$ are reported on samples grown by the molecular beam epitaxy technique, with current-carrying contacts, acting as source and drain, and two probes extending into the two-dimensional electron gas (2DEG) of the AlGaAs/GaAs quantum well, in the range 77-295 $\mathrm{K}$ for frequencies of $10 \mathrm{~Hz}$ to $1 \mathrm{MHz}$. The time constants are almost independent of temperature and the current dependence is close to linear. The noise is interpreted as Lorentzian-modulated shot noise of the 2DEG current. (C) 1999 American Institute of Physics. [S0021-8979(99)08323-1]
\end{abstract}

\section{INTRODUCTION AND PROPERTIES OF THE SAMPLES}

The samples reported on in this study were grown by molecular beam epitaxy (MBE) at the Eindhoven University of Technology. Both the top layout ( $x-y$ directions) and the layer configuration ( $z$ direction) differ considerably from those of regular (gated or nongated) two-dimensional (2D) electron-gas field-effect transistor (TEGFET) devices. The source-drain length $L$ is rather large $(1000 \mu \mathrm{m})$ and the width $w$ is small $(260 \mu \mathrm{m})$, resulting in high-resistance devices. Since $R=L /\left(w n_{s} \mu e\right)$, where $n_{s}$ is the sheet carrier density $\left(\approx 2.3 \times 10^{11} \mathrm{~cm}^{-2}\right)$ and $\mu$ is the mobility (7510 $\mathrm{cm}^{2} / \mathrm{V} \mathrm{s}$ at room temperature), the resulting resistance is about $14 \mathrm{k} \Omega$, decreasing to close to $1 \mathrm{k} \Omega$ at liquid- $\mathrm{N}_{2}$ temperature. The current-voltage characteristics were linear in all cases. The top view of these "Hall structures" is given in Fig. 1.

The Hall data yielded the values of the electron density $n_{s}$, which are constant below $80-100 \mathrm{~K}$, as pictured for samples W228-1 and W228-2 in Fig. 2. Some resistance data are plotted in Fig. 3. The measurement results in the temperature range from 78 to $295 \mathrm{~K}$ show that there is a shift around $200 \mathrm{~K}$. The mobility results of Fig. 4 indicate a complete absence of impurity scattering up to $40 \mathrm{~K}$. At low temperatures $(\leqslant 40 \mathrm{~K})$ the mobility reaches values of $10^{6} \mathrm{~cm}^{2} / \mathrm{V}$ s. Above $40 \mathrm{~K}$ the mobility decreases with $T^{-x}$, $x \approx 2.2$ as expected for polar optical phonon scattering in GaAs. ${ }^{1}$

As to the layer configuration we note that the doped AlGaAs layer is approximately $380 \AA$, whereas the neutral spacer is considerably thicker than in conventional structures, viz., $600 \AA$; therefore, parallel conduction through the doped AlGaAs layer is negligible and it can be assumed that

\footnotetext{
a)Electronic mail: vanvliet@solix.fiu.edu
}

the current flows mainly in the quasi-2D quantum well interface plane. The adjacent GaAs buffer layer can be treated as an insulator, similar to the substrate. Two types of contacts were employed. Sample W228-1 had AuGeNi-alloy contacts formed by standard techniques, whereas sample W228-2 was provided with Sn contacts. Pellets were put on the sample and diffused downward at $450{ }^{\circ} \mathrm{C}$, providing $n^{+}$ contacts to the two-dimensional electron gas (2DEG). The noise of the Sn-contact sample was found to be at least two orders of magnitude less than for the AuGeNi-alloy contacts, for both the 1/f and Lorentzian noise contributions, see below.

\section{EXPERIMENTAL SETUP}

Noise measurements were performed as a function of temperature $T$ for $78 \mathrm{~K} \leqslant T \leqslant 300 \mathrm{~K}$ and frequency $f$ for $10 \mathrm{~Hz} \leqslant f \leqslant 1 \mathrm{MHz}$. A brass box was made, containing the front-end circuitry and bias supply in order to shield the input circuit from outside signals. Calibration was achieved with a white-noise generator, Quan-Tech 420B $(10 \mathrm{~Hz}-100$ $\mathrm{kHz}$ ) and a self-built noise generator based on an avalanche diode and integrated-circuit differential amplifier $(10 \mathrm{kHz}-4$ $\mathrm{MHz}$ ). During the experiments the Hall structure device was placed in a Janis nitrogen bath cryostat with its temperature controlled by a Cryotronics VPF-100 autotuning thermal controller. The cryostat also acted as an external noise shield.

A Brookdeal 5184 low-noise amplifier was used to amplify the signals generated by the noise generator and the device under test. The spectral intensity of the output of the Brookdeal amplifier was measured by a HP3589A spectrum analyzer interfaced with a computer, which provided postaveraging, data storage, and a $\log -\log$ display. Stable averages of $\leqslant 5 \%$ standard derivation were obtained. A flow chart of the measurement procedure is given in Fig. 5.

The device under test (DUT) can be represented by a current generator expressing the excess noise in parallel with 


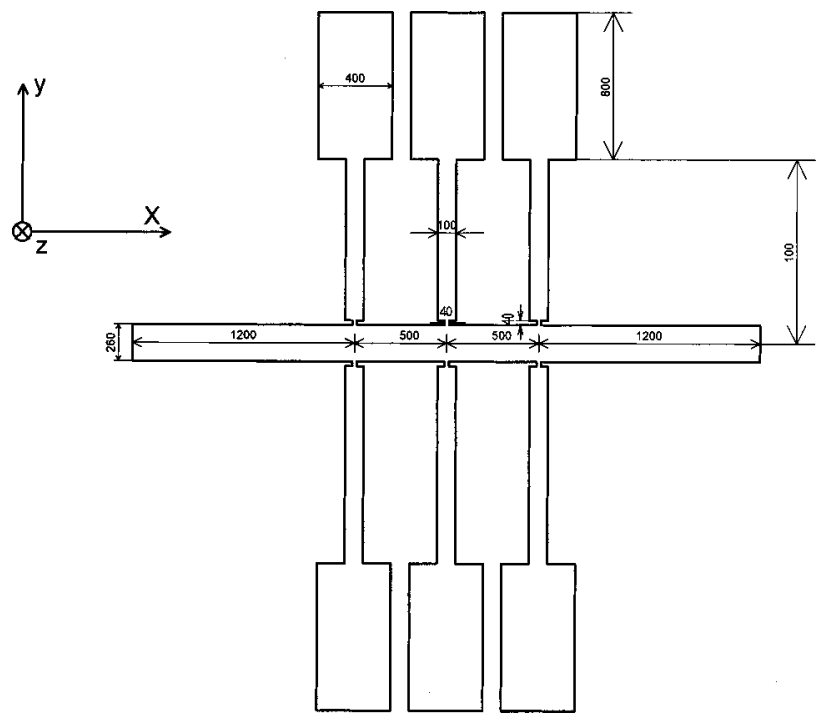

FIG. 1. Top view of the Hall structure sample.

the small-signal resistance of the device itself. The low-noise amplifier is modeled as a noiseless amplifier with a voltage gain, a voltage noise source $S_{V \text { eq }}$ in series to the input and a current source $S_{\text {Ieq }}$ in parallel to the input. The noiseequivalent scheme of the experimental setup is shown in Fig. 6 . Here, $R_{x}$ is the device resistance between the probes, $R_{L}^{\prime}$ represents the part of the device between the probes and external contacts, and $R_{L}$ is the load resistance proper. Two coils were inserted $\left(\omega L \ll R_{L}\right)$ to avoid pickup in the floating leads.

The calibration resistance $R_{s}$, the load impedance of the device $R_{1}=(1 / 4)\left(R_{L}+R^{\prime}{ }_{L}\right)$, and the device resistance $R_{\mathrm{DUT}}=R_{x}$ can be put together into an equivalent input resistance $R_{\text {in }}=R_{x}\left\|R_{1}\right\| R_{s}$ representing an equivalent current source $4 k T / R_{\text {in }}$. The input impedance $Z_{\text {in }}$ is $R_{\text {in }} \|\left(1 / j \omega C_{\text {in }}\right)$ $=R_{\text {in }} /\left(1+j \omega C_{\text {in }} R_{\text {in }}\right)$. The output of the analyzer can be expressed in terms of the power spectral densities of the noise sources. Capacitive shunting is considerable for these highOhmic devices, but canceled out by the measurement of $S_{I}(\omega)$ rather than $S_{V}(\omega)$, as set forth below.

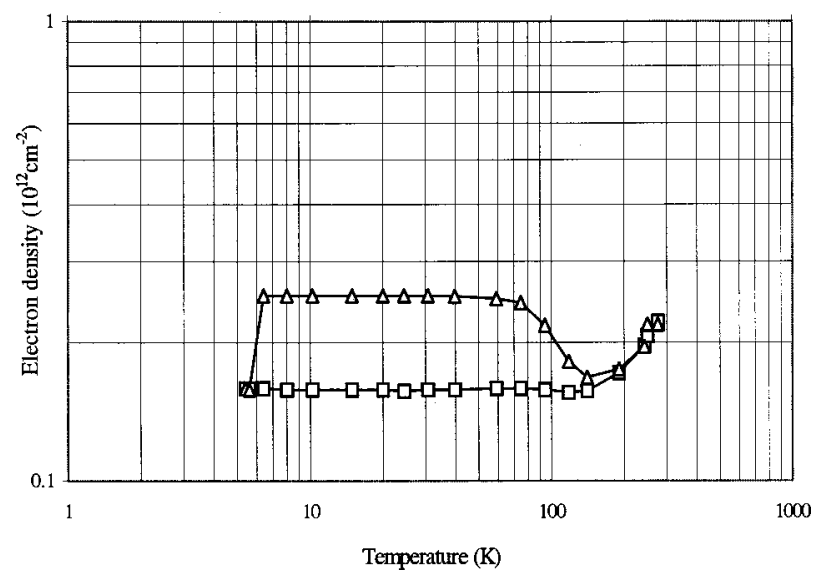

FIG. 2. Electron density vs temperature at $B=0.5 \mathrm{~T}, I=10 \mu \mathrm{A}$. $\square: n_{s}$ for sample W228-1 and $\triangle: n_{s}$ for sample W228-2.

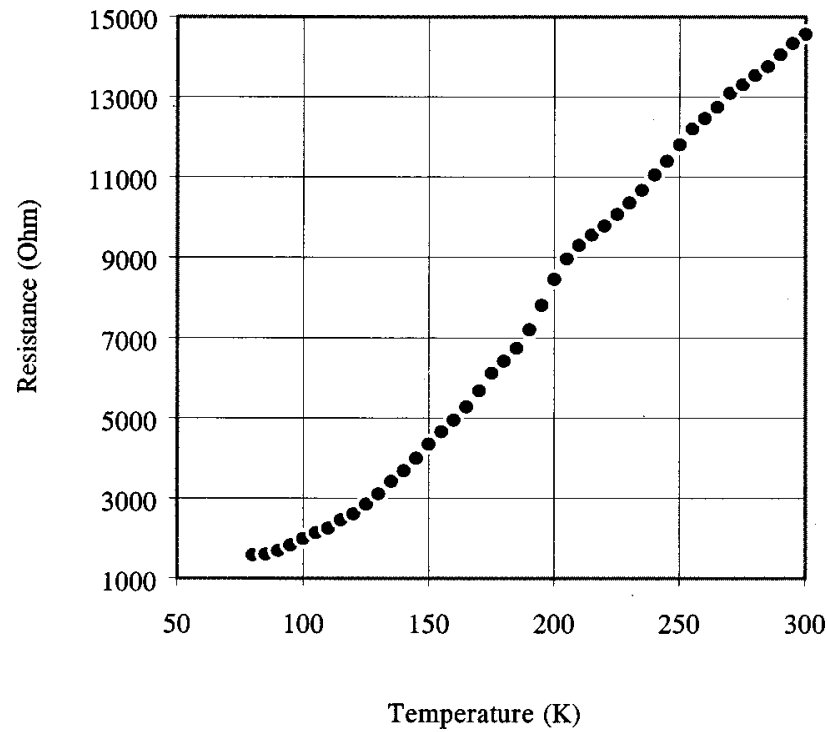

FIG. 3. Resistance vs temperature for sample W228-2.

The noise measurement procedure is the three-point measurement technique. Let $M_{1}$ be the output analyzer reading with a current flowing through the device under test and the noise generator turned off; $M_{2}$ be the output analyzer reading with the noise calibration source applied to the terminal without current flowing through the device under test and the noise generator set to a value $V_{n}$ per $\sqrt{\mathrm{Hz}}$; and $M_{3}$ be the output analyzer reading without current flowing through the device under test and the noise generator (NG) off. Then, noting there are four Norton generators in parallel to $Z_{\text {in }}$

$$
M_{1}=X\left\{\left|Z_{\text {in }}\right|^{2}\left[S_{I, \text { DUT }}(f)+\frac{4 k T}{R_{\text {in }}}+S_{I \mathrm{eq}}(f)\right]+S_{V \mathrm{eq}}\right\},
$$

where $X$ is a constant resulting from the amplification and the bandwidth of the system. Further,

$$
M_{2}=X\left\{\left|Z_{\text {in }}\right|^{2}\left[\frac{S_{V, \mathrm{NG}}}{R_{s}^{2}}+\frac{4 k T}{R_{\text {in }}}+S_{\text {Ieq }}(f)\right]+S_{V \mathrm{eq}}\right\},
$$

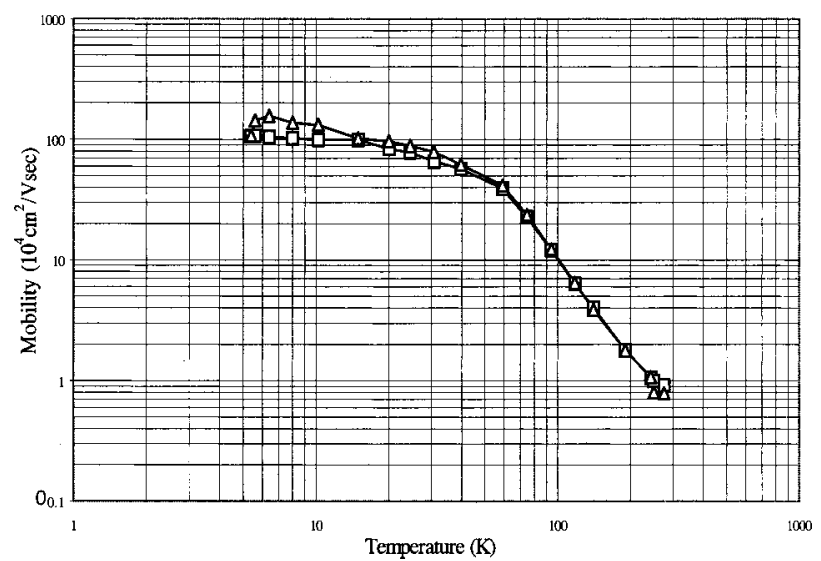

FIG. 4. Mobility vs temperature at $B=0.5 \mathrm{~T}, I=10 \mu \mathrm{A}$. $\square: n_{s}$ for sample W228-1 and $\triangle: n_{s}$ for sample W228-2. 


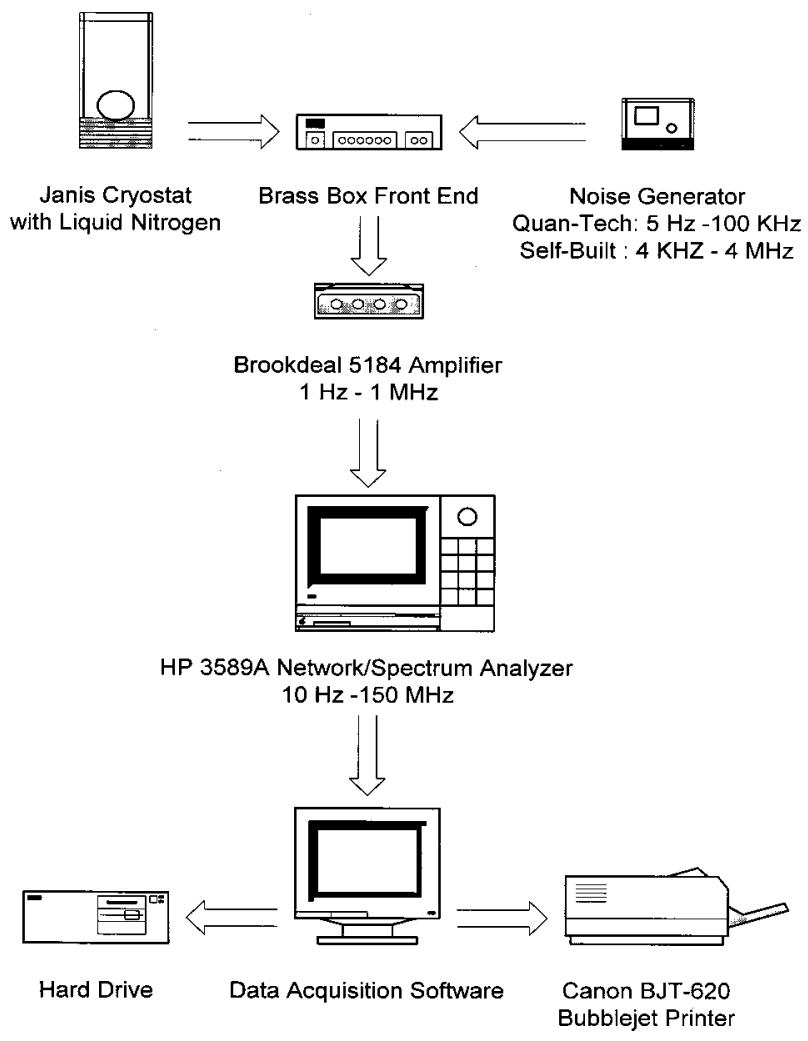

FIG. 5. Measurement procedure.

where $S_{V, \mathrm{NG}} /\left(R_{S}\right)^{2}=S_{I, \mathrm{NG}}, S_{V, \mathrm{NG}}=\left(V_{n}\right)^{2}$ is the voltage intensity output of the calibration noise generator in a bandwidth of $1 \mathrm{~Hz}$. Finally,

$$
M_{3}=X\left\{\left|Z_{\text {in }}\right|^{2}\left[\frac{4 k T}{R_{\text {in }}}+S_{\text {Ieq }}(f)\right]+S_{V \mathrm{eq}}\right\} .
$$

It follows that

$$
S_{I, \mathrm{DUT}}(f)=\frac{M_{1}-M_{3}}{M_{2}-M_{3}} \frac{S_{V, \mathrm{NG}}}{R_{S}^{2}} .
$$

Note that our $S_{I, \text { DUT }}$ includes only “excess noise," i.e., noise over and above the thermal noise.

\section{EXPERIMENTAL RESULTS}

The noise measurements were made in the Ohmic range with probes to eliminate the contribution of the contact

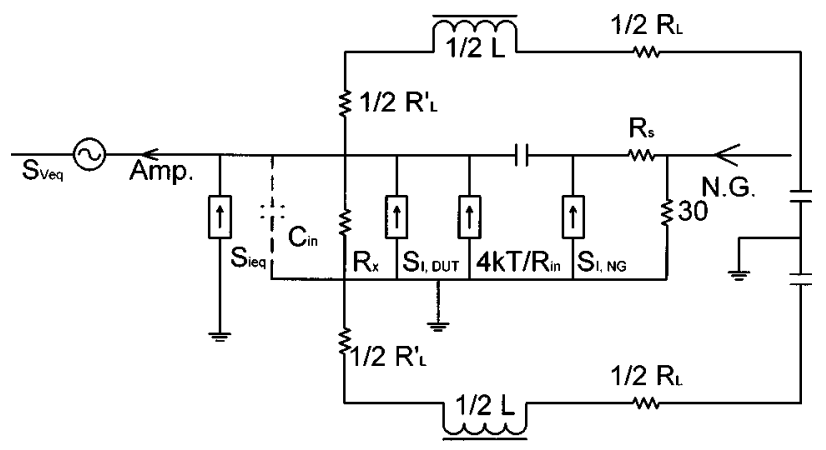

FIG. 6. Equivalent scheme of the experimental setup.

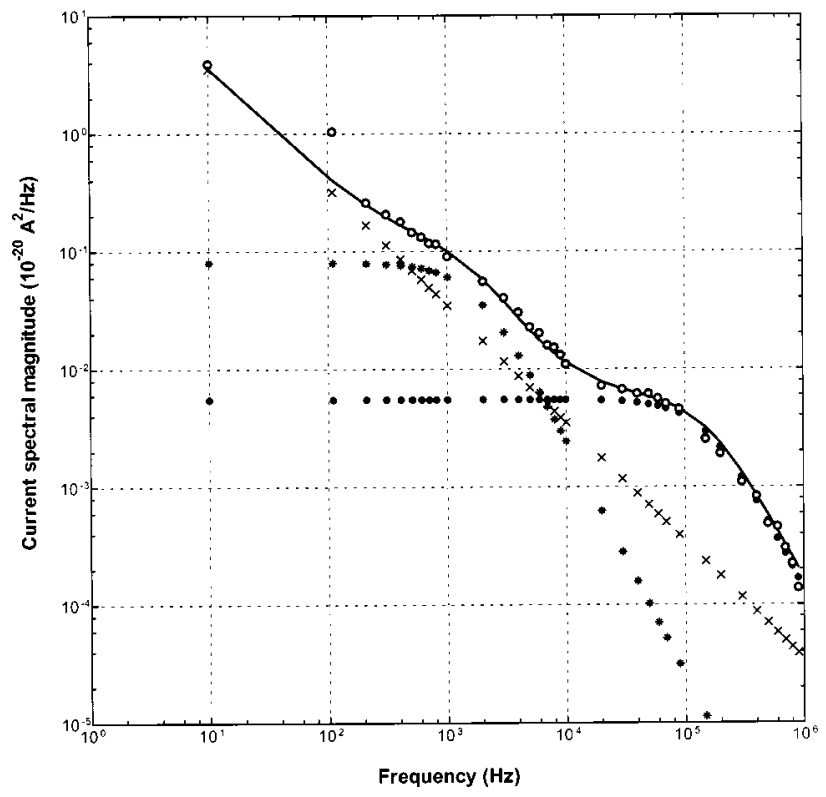

FIG. 7. Current spectrum for sample W228-1 at $T=297 \mathrm{~K}, I=100 \mu \mathrm{A}$. The circles $\bigcirc$ represent the measurement data. The solid line shows the envelope of the $1 / f$ noise plus two or three Lorentzian components, while the partial spectra are given by symbols $\times,+, *$, and $\bullet$ (in this curve the Lorentzian with + is absent). The best-fit curve was obtained by using Microsoft EXCEL software.

noise. A typical current spectral density is shown in Fig. 7, which is for sample W228-1 at $297 \mathrm{~K}$ and $100 \mu \mathrm{A}$. Each of the spectra has two or three Lorentzians in addition to $1 / f$ noise. The spectra are fitted by the form

$$
S_{I}(\omega)=\frac{A}{f^{\alpha}}+\left(\frac{B}{1+\omega^{2} \tau_{1}^{2}}\right)+\frac{C}{1+\omega^{2} \tau_{2}^{2}}+\frac{D}{1+\omega^{2} \tau_{3}^{2}},
$$

[the () on the second term indicates that this very lowfrequency Lorentzian is sometime absent]. Figure 8 gives another current spectrum for W228-1 at lower temperature, $222 \mathrm{~K}$, and $100 \mu \mathrm{A}$. Figures 9 and 10 are two current spectra

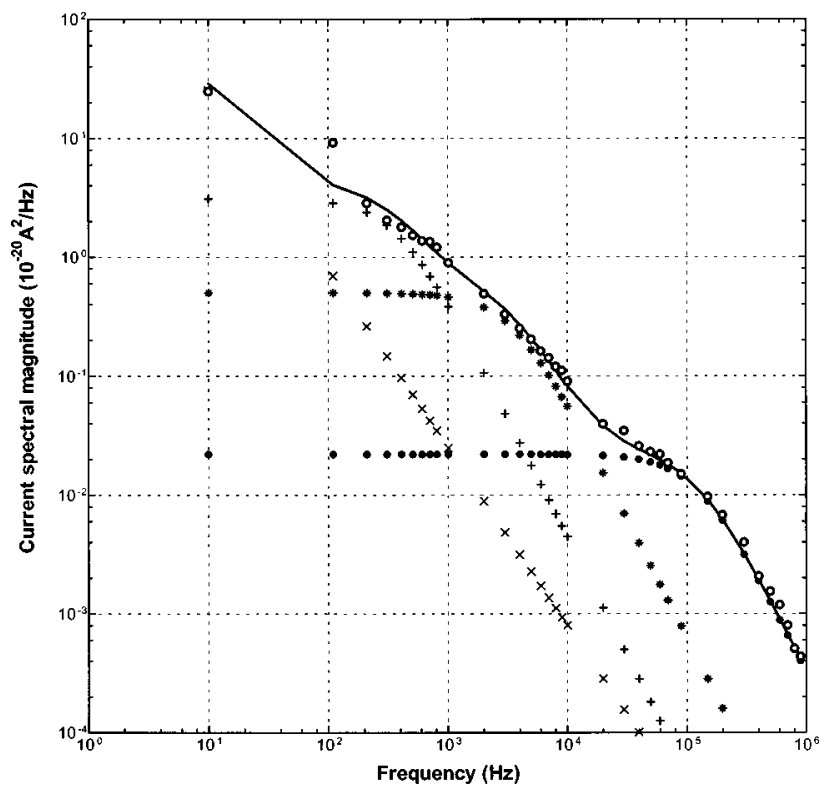

FIG. 8. Current spectrum for sample W228- 1 at $T=222 \mathrm{~K}, I=100 \mu \mathrm{A}$. 


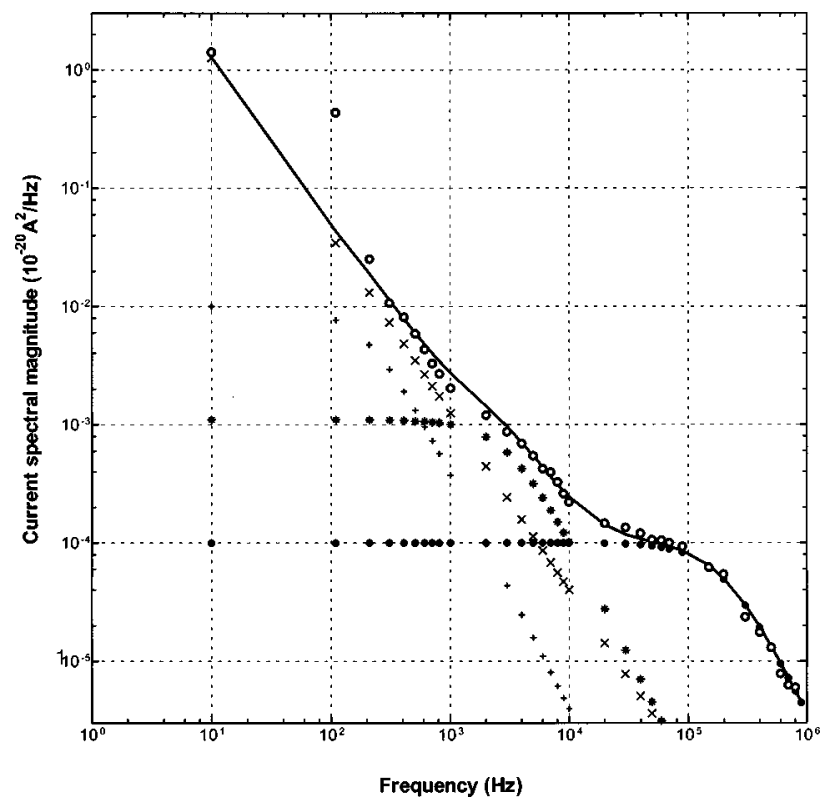

FIG. 9. Current spectrum for sample W228-2 at $T=154 \mathrm{~K}, I=300 \mu \mathrm{A}$.

for sample W228-2 at 154 and $78 \mathrm{~K}$, respectively. Table I gives the measured data of W228-2, the Sn-contact sample. It clearly shows that the overall spectra vary little over the measured temperature range. This fact also holds for W228-1, the AuGeNi-alloy contact sample. It is also noticed that the noise is strongly affected by the nature of the source and/or the drain contacts, with the Sn contacts exhibiting far lower noise (two orders of magnitude) than the AuGeNialloy contacts; furthermore, the noise does not have the usual $I^{2}$ dependence but has a close to a linear current dependence, as shown in Fig. 11. The above observations are the basis for the interpretation in the next section.

\section{TENTATIVE INTERPRETATION}

In contrast to all previously reported data on AlGaAs/ GaAs devices ${ }^{2-4}$ - and also at variance with another paper ${ }^{5}$ - the noise cannot be interpreted as $g-r$ noise of the AlGaAs layer since (a) the time constants do not reveal the presence of activation energies and (b) the variation with current is close to linear. We are, therefore, led to believe that the noise of these samples truly originates in the 2D electron gas of the well and that it should be interpreted as Lorentzian-modulated shot noise, associated with emission centers formed by the interface states in the vicinity of the internal source (or drain) contact.

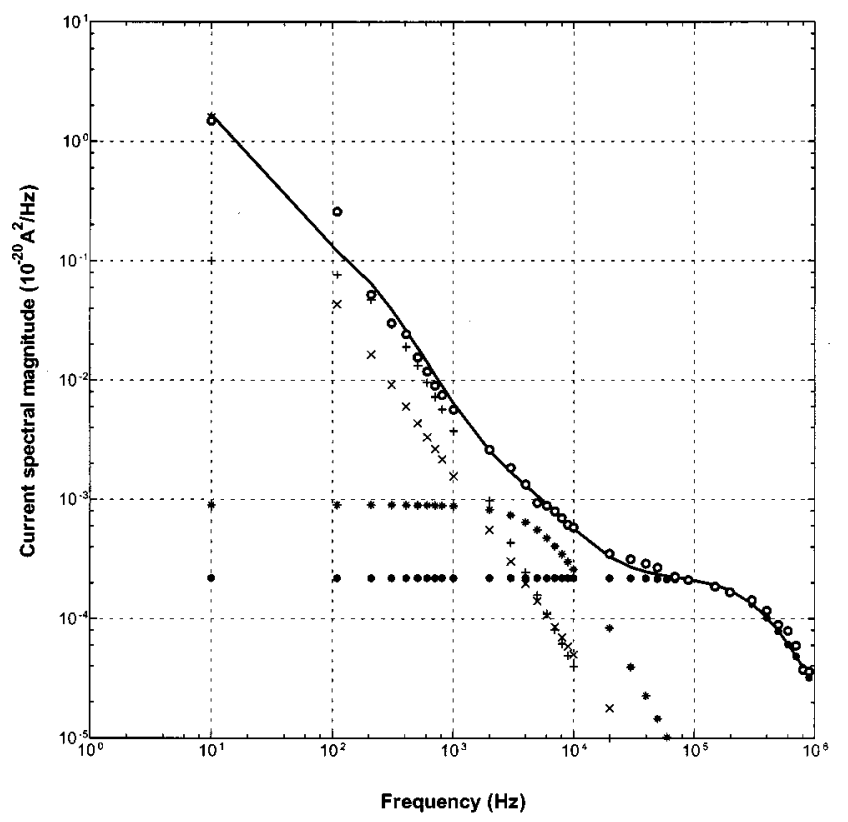

FIG. 10. Current spectrum for sample W228-2 at $T=78 \mathrm{~K}, I=500 \mu \mathrm{A}$.

The discrete energy levels of the well, $\epsilon_{0}, \epsilon_{1}, \epsilon_{2}, \ldots$, give rise to subband contributions in the $x-y$ plane, see Fig. 12. Due to the "logarithmic Fermi statistics" for the surface densities, ${ }^{6}$

$$
n_{i s}=N_{c}^{(2)} k T \ln \left\{1+\exp \left[\left(\varepsilon_{F}-\varepsilon_{i}\right) / k T\right]\right\},
$$

where $i=0,1,2, \ldots$, refers to the subbands, and $N_{c}^{(2)}$ $=2\left(2 \pi m^{*} k T / h^{2}\right)$ is the two-dimensional density of states, the temperature dependence of $n_{i s}$ is weak. Whereas the subbands interchange carriers due to optical or intervalley phonon transfer (see below), these transitions have relaxation times of roughly $1 \mathrm{ps}$; so, for our frequency range the occupancies are stationary, although subject to low-frequency fluctuations, due to injection and/or extraction at the source and/or drain. A plot of the occupancies $n_{0 s}(T)$ and $n_{1 s}(T)$ is given in Fig. 13 for the range 78-300 K. Due to the logarithmic Fermi statistics of Eq. (6), there is only a weak temperature dependence [in complete contrast to a threedimensional (3D) gas]; $n_{0 s}$ changes by $12 \%$ and $n_{1 s}$ by a factor of approximately 6 . Intersubband transitions do occur, but since the energy separation is of order $35 \mathrm{meV}$, only polar optical phonons and intervalley phonons can mediate the electron transfer; we note that for GaAs $\hbar \omega_{\text {polar opt. }}=37 \mathrm{meV}$ and $\hbar \omega_{\text {intervalley }}=30 \mathrm{meV}$, see Ref. 7 . With a mean-free path of $1000 \AA$ and thermal velocity of $10^{7}$

TABLE I. Current spectrum $S_{I}(\omega)$ data for sample W228-2.

\begin{tabular}{|c|c|c|c|c|c|c|c|c|c|}
\hline $\begin{array}{c}T \\
(\mathrm{~K})\end{array}$ & $\begin{array}{c}I \\
(\mu \mathrm{A})\end{array}$ & $\alpha$ & $\begin{array}{c}A \\
\left(10^{-20}\right) \\
\left(\mathrm{A}^{2} / \mathrm{Hz}\right)\end{array}$ & $\begin{array}{c}B \\
\left(10^{-20}\right) \\
\left(\mathrm{A}^{2} / \mathrm{Hz}\right)\end{array}$ & $\begin{array}{c}\tau_{1} \\
\left(10^{-4}\right) \\
(\mathrm{s})\end{array}$ & $\begin{array}{c}C \\
\left(10^{-20}\right) \\
\left(\mathrm{A}^{3} / \mathrm{Hz}\right)\end{array}$ & $\begin{array}{c}\tau_{2} \\
\left(10^{-5}\right) \\
(\mathrm{s})\end{array}$ & $\begin{array}{c}D \\
\left(10^{-20}\right) \\
\left(\mathrm{A}^{2} / \mathrm{Hz}\right)\end{array}$ & $\begin{array}{c}\tau_{3} \\
\left(10^{-7}\right) \\
(\mathrm{s})\end{array}$ \\
\hline 300 & 200 & 1.0 & 0.9 & & & $0.3 \times 10^{-3}$ & 3.3 & $0.5 \times 10^{-4}$ & 8.9 \\
\hline 222 & 250 & 1.0 & 2.8 & $1.5 \times 10^{-2}$ & 4.6 & $2.9 \times 10^{-3}$ & 2.8 & $1.3 \times 10^{-4}$ & 8.5 \\
\hline 154 & 300 & 1.5 & $4.0 \times 10^{1}$ & $1.0 \times 10^{-2}$ & 8.0 & $1.1 \times 10^{-3}$ & 5.0 & $1.0 \times 10^{-4}$ & 8.2 \\
\hline 100 & 400 & 1.5 & $3.0 \times 10^{1}$ & $6.0 \times 10^{-2}$ & 8.0 & $1.1 \times 10^{-3}$ & 1.5 & $3.2 \times 10^{-4}$ & 6.5 \\
\hline 78 & 500 & 1.5 & $5.0 \times 10^{1}$ & $10.0 \times 10^{-2}$ & 8.0 & $0.9 \times 10^{-3}$ & 2.5 & $2.2 \times 10^{-4}$ & 4.3 \\
\hline
\end{tabular}




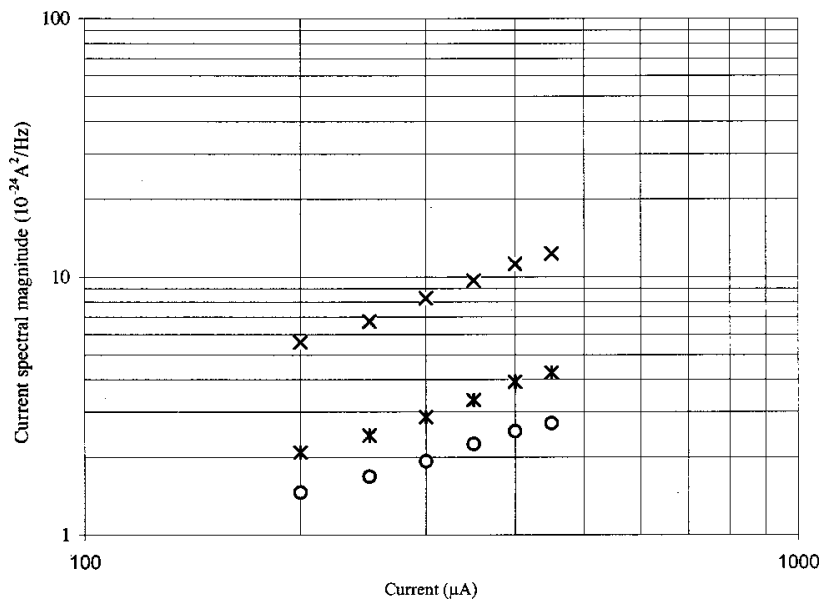

FIG. 11. Spectral values vs current for sample W228-2.

$\mathrm{cm} / \mathrm{s}$ (estimated since $m^{*}$ is not well known), the collision time is of order $1 \mathrm{ps}$. So, the Lorentzians of these processes have turn over frequencies of order $160 \mathrm{GHz}$, entirely outside the scope of our present considerations.

We must, therefore, look to the coupling of the internal source contact to the subbands for the origin of the noise. This is also indicated experimentally; the Sn contacts (sample W228-2) yield two orders of magnitude smaller plateau values than the AuGeNi contacts. Of course, perfect contacts give an unimpeded injection into the 2D $x-y$ plane and produce no noise. We thus assume that part of the injection process takes place via interface states at the internal source contact, which emit their charges via tunnel processes into the subbands. These interface states probably form a continuum on the energy scale, but only states being near the Fermi energy need to be considered. We denote the occupancy per $\mathrm{cm}^{2}$ by $N_{s}$, the total interface density near $\epsilon_{F}$ being $\hat{N}_{s}$. (In noise analysis we need occupancy and state numbers for the statistical distributions whereas densities are used in the kinetic equations. To avoid a double set of symbols we assume, where necessary, a ficticious area of $1 \mathrm{~cm}^{2}$.) The carriers in these states fluctuate, because of random supply by the external source contact (electrode), which acts as an infinite reservoir. We thus assume Poissonian statistics for the filling of the interface states, whereas the coupling to the subbands involves a gain-loss process with exponential delay, see Eq. (10) below.

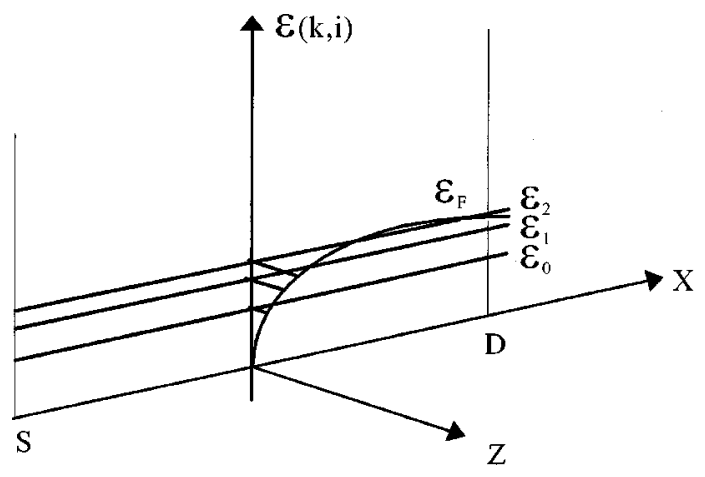

FIG. 12. Structure of the subbands.
The emission process can, therefore, be described as a compound Poisson process, i.e., the probability for the emission of $M$ electrons in an interval $(t, t+\theta)$ at an average rate $\lambda$ is given as

$$
\begin{aligned}
W(M, \theta)= & \sum_{N_{s}} \frac{\left[\lambda \int_{t}^{t+\theta} N_{s}\left(t^{\prime}\right) d t^{\prime}\right]^{M}}{M !} \\
& \times \exp \left\{-\lambda \int_{t}^{t+\theta} N_{s}\left(t^{\prime}\right) d t^{\prime}\right\} P\left(N_{s}\right),
\end{aligned}
$$

where $P\left(N_{s}\right)$ is the stationary probability distribution for the interface occupancy $N_{s}(t)$. The first two factorial moments and the variance are easily obtained; using the overhead bar for averages involving $M$ and angular brackets for averages concerning $N_{s}$, we have

$\bar{M}_{\theta}=\sum_{M} W(M, \theta)=\left\langle\lambda \int_{t}^{t+\theta} N_{s}\left(t^{\prime}\right) d t^{\prime}\right\rangle=\lambda \theta\left\langle N_{s}\right\rangle$,

$\overline{\Delta M_{\theta}^{2}}=\lambda \theta\left\langle N_{s}\right\rangle+\lambda^{2} \int_{0}^{\theta} \int_{0}^{\theta} d t_{1} d t_{2}\left\langle\Delta N_{s}\left(t_{1}\right) \Delta N_{s}\left(t_{2}\right)\right\rangle$.

In the latter expression the correlation function for the interface occupancies occurs, for which we assume

$$
\left\langle\Delta N_{s}\left(t_{1}\right) \Delta N_{s}\left(t_{2}\right)\right\rangle=\left\langle\Delta N_{s}^{2}\right\rangle e^{-\left|t_{2}-t_{1}\right| / \tau} .
$$

Equations (9) and (10) lead to "superstatistical emission noise,' the theory of which was worked out before by Van Vliet, Handel, and van der Ziel. ${ }^{8}$ Via MacDonald's theorem, see the Appendix, one obtains for the spectrum of the flux variations $\Delta m(t)$ of emitted particles

$$
S_{\Delta m}(\omega)=2 \bar{m}\left[1+2 \frac{\left\langle\Delta N_{s}^{2}\right\rangle}{\left\langle N_{s}\right\rangle} \lambda \tau \frac{1}{1+\omega^{2} \tau^{2}}\right],
$$

where we used $\lambda\left\langle N_{s}\right\rangle=\bar{M}_{\theta} / \theta=\bar{m}$ cf., Eq. (8). Multiplying Eq. (11) by $e^{2}$, this gives the current noise. The first term is then the shot noise which is suppressed in a nonballistic device (as shown by Brillouin ${ }^{9}$ ), and will be omitted. The second term is the Lorentzian contribution.

We are now more specific with regards to the gain-loss process of the emission centers. Let $\ell_{i}(t)$ be the loss due to emission into the subband " $i$ "' and $g_{i}$ the gain; clearly, then,

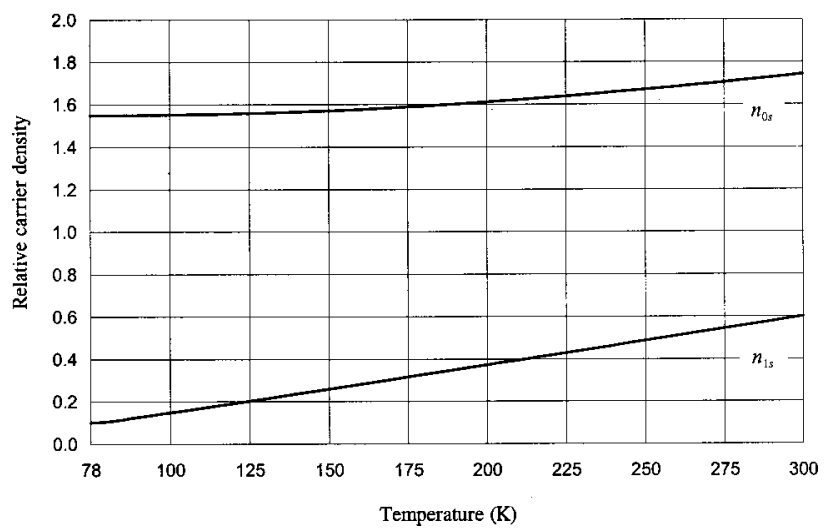

FIG. 13. Relative subband carrier densities vs temperature, showing $n_{0 s}(T)$ and $n_{1 s}(T)$. 


$$
\ell_{i}(t)=\lambda_{i} N_{s}(t), \quad g_{i}(t)=\delta\left[\hat{N}_{s}-N_{s}(t)\right] n_{i s}(t),
$$

where, as stated before, $N_{s}$ is the interface occupancy, $\hat{N}_{s}$ is the total available number, and $n_{i s}$ is the subband occupancy. From the theory of gain-loss ${ }^{10,11}$ processes one finds

$$
\tau_{i}=1 /\left[\ell_{i}^{\prime}\left(N_{s}\right)-g_{i}^{\prime}\left(N_{s}\right)\right]_{\left\langle N_{s}\right\rangle}=1 /\left[\lambda_{i}+\delta\left[\left(\left\langle n_{i s}\right\rangle+\alpha \hat{N}_{s}\right)\right],\right.
$$

where $\alpha$ is the fraction of empty interface states, while $\delta$ $=\left\langle\mathrm{v}_{\mathrm{th}}\right\rangle \sigma_{t} / w$, where $\mathrm{v}_{\mathrm{th}}$ is the thermal velocity, $\sigma_{t}$ the capture cross section of the interface states, and $w$ the width of the well. As in most trapping processes we assume $\hat{N}_{s}$ $-\left\langle N_{s}\right\rangle=\alpha \hat{N}_{s} \ll\left\langle n_{i s}\right\rangle$. Thus, approximately,

$$
\tau_{i} \approx 1 /\left[\lambda_{i}+\delta\left\langle n_{i s}\right\rangle\right] \text {. }
$$

Since now $\lambda_{i}=\ell_{i 0} /\left\langle N_{s}\right\rangle$ [see Eq. (12)] and $\ell_{i 0}=g_{i 0}$ due to detailed balance, we have

$\lambda_{i} \tau_{i}=\frac{g_{i 0} \tau_{i}}{\left\langle N_{s}\right\rangle}=\frac{\delta\left[\hat{N}_{s}-\left\langle N_{s}\right\rangle\right]\left\langle n_{i s}\right\rangle}{\delta\left[\left\langle n_{i s}\right\rangle+\lambda_{i} / \delta\right]\left\langle N_{s}\right\rangle}=\frac{\alpha}{\beta} \frac{\left\langle n_{i s}\right\rangle}{\left\langle n_{i s}\right\rangle+\lambda_{i} / \delta}$,

where $\alpha$ is the fraction of empty interface states and $\beta$ $=\left\langle N_{s}\right\rangle /\left\langle\hat{N}_{s}\right\rangle$ is the fraction of occupied states. Let $I_{i}$ now be the current that passes through the interface states into the subband $i$. Clearly, $I_{i}(t)=e m_{i}(t)$. We obtain from Eqs. (11) and (15), denoting the resulting channel current by $I_{1}$,

$$
\begin{aligned}
S_{\Delta I_{1}}(\omega) & =4 e\left(\frac{\alpha}{\beta}\right) \sum_{i} \bar{I}_{i} \frac{\left\langle n_{i s}\right\rangle}{\left\langle n_{i s}\right\rangle+\lambda_{i} / \delta} \frac{1}{1+\omega^{2} \tau_{i}^{2}} \\
& =4 e\left(\frac{\alpha}{\beta}\right) \sum_{i} \bar{I}_{i}\left\langle n_{i s}\right\rangle \delta \frac{\tau_{i}}{1+\omega^{2} \tau_{i}^{2}} .
\end{aligned}
$$

Finally, we must find a relationship with the total current mitigated and nonmitigated by the interface states-denoted as $I_{0}$. We have

$$
\bar{I}_{i}=e \lambda_{i}\left\langle N_{s}\right\rangle=e \lambda_{i} \beta \hat{N}_{s},
$$

and we write

$$
e \lambda_{i}\left\langle n_{i s}\right\rangle=\chi_{i} I_{1}=\chi_{i} p I_{0},
$$

where $\chi_{i}$ pertains to the fraction of carriers traveling in the subband $i$; further, $p$ denotes the fraction of current mitigated by the interface states. We further write $S_{\Delta I 1}(\omega)=S_{\Delta I}(\omega)$, since no noise-except strongly suppressed shot noise ${ }^{9}$-is attributed to the current fraction $(1-p) I_{0}$. Then, from Eq. (16) and the expression for $\delta$ given after Eq. (13):

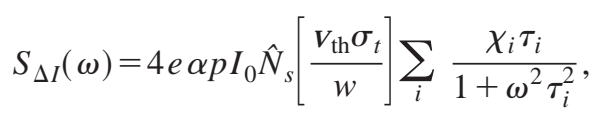

which is the basic form for Lorentzian-modulated shot noise.

Finally, assuming that the emission tunnel process is elastic, note that $\chi_{i} \hat{N}_{s} \equiv N_{i s}$ relates to interface states having an energy within a range $\sim 2 \mathrm{kT}$ of the subband $\epsilon_{i}$. We then have, alternatively,

$$
S_{\Delta I}(\omega)=4 e p I_{0}\left[\frac{\mathrm{v}_{\mathrm{th}} \sigma_{t}}{w}\right] \sum_{i} \alpha_{i} N_{i s} \frac{\tau_{i}}{1+\omega^{2} \tau_{i}^{2}} .
$$

In this form the result is identical with a second model, developed in Ref. 12, in which a priori, the different Lorentz- ians were attributed to different groups of interface states. Without further technological studies no definite distinction between these models can be made, so in this article we focus on the model implied by Eq. (19) for the interpretation.

Thus, since $\left\langle n_{0 s}\right\rangle>\left\langle n_{1 s}\right\rangle>\left\langle n_{2 s}\right\rangle$, we have $\tau_{0}<\tau_{1}<\tau_{2}$; we, therefore, attribute the highest frequency Lorentzianbeing in all spectra the main Lorentzian - to subband $\epsilon_{0}$, the next highest frequency Lorentzian to subband $\epsilon_{1}$, and the lowest frequency Lorentzian to subband $\epsilon_{2}$. The observed plateau values vary $\propto \chi_{i} \tau_{i}$, the prefactor being the same for all Lorentzians, indicating a common origin. Most important, the noise is proportional to $\hat{N}_{s}$, the interface states sheet density. Thus, as we noted already, $\hat{N}_{s}$ must be several orders of magnitude less for the sample with the Sn contacts than for the sample with the AuGeNi-alloy contacts.

In conclusion, this theory, while tentative, explains most of the features of the observed noise, both the strong dependence on $\hat{N}_{s}$ and the very weak dependence of the relaxation times on temperature, see Eq. (14) and Fig. 13. For the highest frequency Lorentzian, Table I indicates that $\tau_{3}$ is, by and large, constant, commensurate with $\delta_{0} n_{0} \gg \lambda_{0}$ for most temperatures. For the second Lorentzian $\tau_{2}$, shows the same pattern, suggesting that $\tau_{2} \rightarrow 1 / \lambda_{1}$ as the temperature decreases. Although the temperature dependence of the $\tau_{i}$ is not fully explained in this model, the reader will note that "nearconstant" relaxation times are strongly indicative of 2DEG behavior, and are not possible in any 3D electron gas, in which the occupancies vary over orders of magnitude, leading to Arrhenius plots for $\log \left(\tau T^{2}\right)$, see Refs. 2-5. To obtain some quantitative data we consider the main Lorentzian with $\tau \approx 5 \times 10^{-7} \mathrm{~s}$, representing the gain-loss process for the first subband. From Eq. (14), neglecting $\lambda_{0}$ and assuming $n_{s 0} \approx 10^{11} \mathrm{~cm}^{-2}$, we find $\delta \approx 2 \times 10^{-5} \mathrm{~cm}^{2} / \mathrm{s}$. With $w=3$ $\times 10^{-7} \mathrm{~cm} \quad(30 \AA), \quad \mathrm{V}_{\mathrm{th}}=10^{7} \mathrm{~cm} / \mathrm{s}, \quad$ this yields $\sigma_{t}$ $\approx 10^{-18} \mathrm{~cm}^{2}$, which is an acceptable value for the capture cross section of negatively charged interface states; similar cross sections are reported by Hofman et al. ${ }^{4}$ and elsewhere in the literature.

\section{ACKNOWLEDGMENTS}

This research was sponsored by the Future Aerospace Science and Technology in Cryoelectronics (FAST) center, supported by AF-OSR under Contract No. F49620-95-10519. The equipment was obtained under Contract No. F33615-96-C-5452 with Wright Materials Laboratory WL/ ML, Wright Patterson AFB.

\section{APPENDIX: COMPOUND POISSON SPECTRA}

A compound Poisson distribution was employed by Mande ${ }^{13}$ for the description of wave-interaction noise. Subsequently, Van Vliet, Handel, and Van der Ziel ${ }^{8}$ have shown that "superstatistical emission noise" occurs in a large variety of fluctuation processes, from flicker noise as originally envisioned by Schottky, to cathodoluminescence fluctuations in the light output of phosphors ${ }^{14}$ in which Lorentzians occur due to the time delay of the deactivation of excited emission levels. This phenomenon closely resembles the model proposed here for the source-drain current in the 2DEG. 


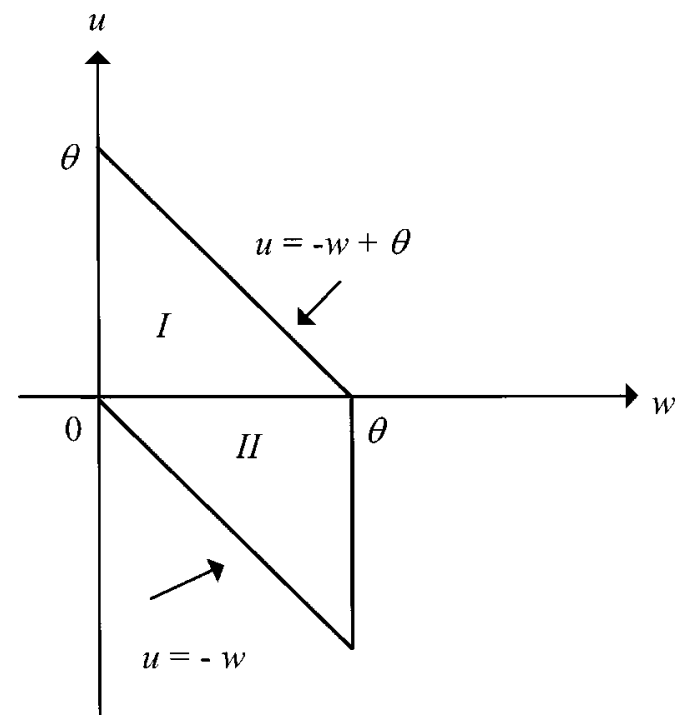

FIG. 14. Evaluation of the integral (A1).

The compound Poisson distribution Eq. (7) easily yields the first two factorial moments. Thus, for the variance,

$$
\begin{aligned}
\overline{\Delta M_{\theta}^{2}}= & \overline{M_{\theta}\left(M_{\theta}-1\right)}-\overline{M_{\theta}}\left(\overline{M_{\theta}}-1\right) \\
= & \overline{M_{\theta}}+\lambda^{2} \int_{t}^{t+\theta} \int_{t}^{t+\theta}\left\langle N_{1}\left(t_{1}\right) N_{s}\left(t_{2}\right)\right\rangle d t_{1} d t_{2} \\
& -\lambda^{2} \int_{t}^{t+\theta}\left\langle N_{1}\left(t_{1}\right)\right\rangle d t_{1} \int_{t}^{t+\theta}\left\langle N_{s}\left(t_{2}\right)\right\rangle d t_{2} \\
= & \lambda \theta\left\langle N_{s}\right\rangle+\lambda^{2} \int_{0}^{\theta} \int_{0}^{\theta}\left\langle\Delta N_{1}\left(t_{1}\right) \Delta N_{2}\left(t_{2}\right)\right\rangle d t_{1} d t_{2} .
\end{aligned}
$$

To obtain the integral subject to the correlation function Eq. (10), we set $t_{1}=t_{2}+u, t_{2}=w$, see Fig. 14. Integrating separately over regions I and II we obtain for region I

$$
\mathfrak{J}_{\mathrm{I}}=\int_{0}^{\theta} d w \int_{0}^{-w+\theta} d u e^{-u / \tau}=\tau \int_{0}^{\theta} d \psi\left(1-e^{-\psi / \tau}\right),
$$

where $\psi=\theta-w$. Likewise for II

$$
\mathfrak{J}_{\mathrm{II}}=\int_{0}^{\theta} d w \int_{-w}^{0} d u e^{u / \tau}=\tau \int_{0}^{\theta} d w\left(1-e^{-w / \tau}\right) .
$$

Thus, the full result becomes

$$
\overline{\Delta M_{\theta}^{2}}=\lambda \theta\left\langle N_{s}\right\rangle+2 \lambda^{2}\left\langle\Delta N_{s}^{2}\right\rangle \tau \int_{0}^{\theta} d w\left(1-e^{-w / \tau}\right) .
$$

Further, let $m$ be the rate of emitted particles in the interval $(t, t+\theta)$. Then, for the finite time average

$$
\begin{aligned}
& m_{\theta}(t)=\frac{1}{\theta} \int_{t}^{t+\theta} m\left(t^{\prime}\right) d t^{\prime}=\frac{M_{\theta}(t)}{\theta}, \\
& \text { and }\left\langle\Delta m_{\theta}^{2}\right\rangle=\frac{1}{\theta^{2}} \overline{\Delta M_{\theta}^{2}} .
\end{aligned}
$$

Using Eq. (A4), this yields

$$
\frac{\partial}{\partial \theta}\left(\theta^{2} \overline{\Delta m_{\theta}^{2}}\right)=\lambda\left\langle N_{s}\right\rangle+2 \lambda^{2}\left\langle\Delta N_{s}^{2}\right\rangle \tau\left(1-e^{-\theta / \tau}\right) .
$$

The spectrum for the flux $m(t)$ is computed from MacDonald's theorem ${ }^{15}$

$$
S_{\Delta m}(\omega)=2 \omega \int_{0}^{\infty} d \theta \sin (\omega \theta) \frac{\partial}{\partial \theta}\left(\theta^{2} \overline{\Delta m_{\theta}^{2}}\right) .
$$

A straightforward calculation yields the main result [Ref. 8, Eq. (2.19)];

$$
S_{\Delta m}(\omega)=2 \lambda\left\langle N_{s}\right\rangle\left[1+2 \frac{\left\langle\Delta N_{s}^{2}\right\rangle}{\left\langle N_{s}\right\rangle} \lambda \tau \frac{1}{1+\omega^{2} \tau^{2}}\right] .
$$

${ }^{1}$ B. R. Nag, Theory of Electrical Transport in Semiconductors (Pergamon, New York, 1972).

${ }^{2}$ F. Pascal, M. De Murcia, G. Lecoy, and L. K. J. Vandamme, Solid-State Electron. 37, 1503 (1994)

${ }^{3}$ F. Hofman, R. J. J. Zijlstra, J. M. Bettencourt De Freitas, and J. C. M. Henning, Solid-State Electron. 34, 23 (1991).

${ }^{4}$ F. Hofman, R. J. J. Zijlstra, and J. C. M. Henning, Solid-State Electron. 31, 279 (1988).

${ }^{5}$ Y. P. Chen, C. M. Van Vliet, G. L. Larkins, Jr., and H. Morkoç, IEEE Trans. Electron Devices (submitted).

${ }^{6}$ M. Shur, GaAs Devices and Circuits (Plenum, New York, 1987).

${ }^{7}$ S. M. Sze, Physics of Semiconductor Devices, 2nd ed. (Wiley, New York, 1981), Appendix H.

${ }^{8}$ K. M. Van Vliet, P. H. Handel, and A. van der Ziel, Physica A 108, 511 (1981).

${ }^{9}$ L. Brillouin, Helv. Phys. Acta 7 (Suppl.), 47 (1934).

${ }^{10}$ R. E. Burgess, Physica (Amsterdam) 20, 1007 (1954).

${ }^{11}$ K. M. Van Vliet and J. R. Fassett, in Fluctuation Phenomena in Solids, edited by R. E. Burgess (Academic, New York, 1965), pp. 267-354.

${ }^{12}$ Y. P. Chen, Ph.D. thesis, Florida International University (1998).

${ }^{13}$ L. Mandel, Proc. Phys. Soc. London 81, 1140 (1963).

${ }^{14}$ H. M. Fijnhaut and R. J. J. Zijlstra, J. Phys. D: Appl. Phys. 3, 45 (1970).

${ }^{15}$ D. K. C. MacDonald, Rep. Prog. Phys. 12, 56 (1948). 